\title{
Is visual information integrated across successive fixations in reading?
}

\author{
GEORGE W. McCONKIE and DAVID ZOLA \\ Center for the Study of Reading, University of Illinois, Champaign, Illinois 61820
}

\begin{abstract}
College students read a passage presented in AlTeRnAtInG cAsE on a CRT while their eye movements were monitored. During certain saccades, the case of every letter was changed (a became A, B became b). This change was not perceived and had no effect on eye movements. Apparently visual features of the type which specify the difference between upper- and lowercase letters are not integrated across fixations during reading.
\end{abstract}

One of the most well-established facts about reading concerns the saccadic nature of eye movements. About four times a second, the eye is cast to a new location, supposedly giving the reader a new glimpse of the test. Although the reader has the experience of passing somewhat smoothly along the line in reading, in fact, the eye is relatively still over $90 \%$ of the time, with movements requiring only 20-40 msec occuring about 4 times/sec (Woodworth, 1938).

This fact about eye movements gives rise to a basic question about reading: How is information from discrete glimpses of the text orchestrated into a smoothly proceeding reading process? How is information integrated across fixations?

The need for some sort of combining of information seems obvious from our ability to perceive a coherent world from such discrete input. However, a study by Rayner (1975) specifically demonstrated the problem in reading. While subjects were reading a paragraph displayed on a computer-controlled cathode-ray tube (CRT) a saccade was identified that was likely to center the eye on a preselected word location. The contents of that word location were then changed during that same saccade. Thus, the word lying in the fovea on one fixation, following the display change, was different from the word which had occupied that location in the text on the prior fixation. If the prior fixation had been within 12 letter positions to the left of the critical word location, the fixation following the change (which was centered on the changed word) was longer than if no change had occurred. This was taken as evidence that the change in stimulus from one fixation to the next had affected the processing that occurs during reading; presumably this caused some disruption of the process by which information from successive fixations is

This research was supported by Grant MH24241 from the National Institute of Mental Health. Requests for reprints should be sent to George W. McConkie, The Center for the Study of Reading, University of Illinois, 51 Gerty Drive, Room 174, Champaign, Illinois 61820 . integrated, thereby inflating the processing time required, and thus lengthening the fixation.

The problem to be addressed in the present paper concerns the nature of the visual information that is carried over from one fixation to the next. One possible explanation would involve some sort of buffer into which visual information is loaded during each fixation, and from which the language processes would draw as needed to support processing. Rayner (1975) and McConkie and Rayner (1976) specifically postulated a buffer of this sort, and labeled it the "integrative visual buffer." They suggested that, even though retinal information may be masked from one fixation to the next, the visual information from successive fixations must be integrated into a single set at some higher level in the system. Cumming (in press) points out the two bases on which visual information from two fixations may be "overlapped" into such a buffer: (1) by knowing where the eye is being sent, thus justifying the two sets of information on this basis (mentally "shifting" the visual data from one fixation a distance corresponding to the length of the saccade before bringing the two sets of data together), or (2) by matching them on the basis of some aspect of their figural properties. McConkie and Rayner (1976) opted for the latter.

The critical factor is that this model assumed that strictly visual information was being brought together from successive fixations and integrated into a single information set. It was assumed that if visual characteristics of the stimulus pattern were changed from one fixation to the next, disruption would occur in the integration process (Rayner, 1975). The goal of the study to be reported here is to further test this assumption.

What is needed to test this assumption is a means of changing strictly visual characteristics of the text from one fixation to the next without modifying other characteristics (orthographic, syntactic, semantic, etc). The best example of such a manipulation would be to change letters from upper to lower case, or vice versa, from one fixation to the next. Thus, while 
the visual features change, the letter sequences and words do not. However, to change a line of text from all upper to lower case represents a rather substantial change in the global figural characteristics of the text. A more subtle change is permitted by having subjects read text printed in AlTeRnAtInG cAsE. An example of a line of text in this format is shown in Figure 1, which also shows the alternative version of that same line in which the case of every letter has been changed. The gross visual appearance of the two lines is quite similar, yet the shape of every letter has been changed from one version to the other (of course, some letters change shape more than others).

It was assumed that if the entire line were changed from one of these versions to the other during a saccade, so that the visual characteristics of every letter and word were different on one fixation than they had been on the last, this would greatly disrupt any visual integration process of the type described above, and would therefore disrupt reading. The experiment to be described was designed to learn whether such a disruption occurs.

\section{METHOD}

\section{Subjects}

Eight upperclass college students who were experienced with eye-movement monitoring procedures were paid to participate in this study.

\section{Materials}

Text: Two 350-word passages were constructed, each containing 30 lines of text, with each line being between 66 and 70 character positions in length. One was a biographical sketch about Harry Houdini, and the other was about Mangrove trees. The text was displayed in alternating case, as shown in Figure 1.

Questions: In order to insure that the subjects read the passages and understood their basic content, five comprehension questions were constructed for each passage. The questions were designed to test general information, detail information, and inferential information.

\section{Procedure}

The texts were displayed one line at a time on a CRT, with a new line appearing each time the subject pressed a button. The subjects read each passage while their eye movements were monitored and recorded by computer (for details of the equipment used, see McConkie, Zola, Wolverton, \& Burns, 1978).

After a brief equipment calibration procedure, each subject read the first passage to become familiar with text printed in alternating case. No display changes were made as they read this passage. After answering the test questions and recalibrating, they read the second passage, which served as the experimental passage. As they read this passage, the computer identified and counted forward saccades in which the eye reached a velocity greater than $185^{\circ} / \mathrm{sec}$. That is, it identified those right-moving saccades which would travel at least about 3.5 letter positions. On half the lines, the computer identified the second, fourth, and sixth saccades meeting this criterion, and on the other half, it identified the third, fifth, and seventh saccades. The computer also marked in the eye-movement data the point at which each of these saccades exceeded the $185^{\circ} / \mathrm{sec}$ threshold. On half the lines, when this threshold was exceeded, on the saccades specified, the version of the text was switched; that is, the case of every letter was changed during the time the eye was moving. The computer also marked the data at the point at which the display change was complete, in order to make sure that the change occurred during the period of the eye movement itself.

The experimental manipulations were completely counterbalanced across subjects. Two subjects read each line under each of four experimental conditions: display changes on Saccades 2, 4, and 6; display changes on Saccades 3,5 , and 7; detection of Saccades 2,4 , and 6 but no display changes; and the same for Saccades 3,5 , and 7 .

After reading the passage, the subjects were administered the retention test.

\section{RESULTS}

The first question is whether the display changes were actually occurring during the time the eye was moving. Since the computer was sampling the reader's eye position every millisecond, it recorded on which millisecond the display change was initiated and on which it was completed. Figure 2 provides an example of the data on a typical eye movement. The display change required only about $3 \mathrm{msec}$ to complete, and it was clear from an examination of the data that in every instance the change occurred well within the period of time that the eye was moving.

Data from the first passage (on which no display changes occurred) indicated that the subjects were able to read text printed in case-alternating format with relative ease, even with little practice. Average fixation duration $(256 \mathrm{msec})$ and average saccade length ( 8.3 letter positions) for the first passage were well within the normal range obtained with conventional print.

Three aspects of the eye-movement data were analyzed to determine whether the display change produced a disruption in reading: durations of fixations, lengths of forward saccades, and number of regressive movements. These were compared for fixations and saccades immediately following the display changes, and for other fixations and saccades on the line. In each case, a comparison was made between the data for lines on which the display changes occurred and corresponding data for lines with no display changes. These data are summarized in Table 1. 


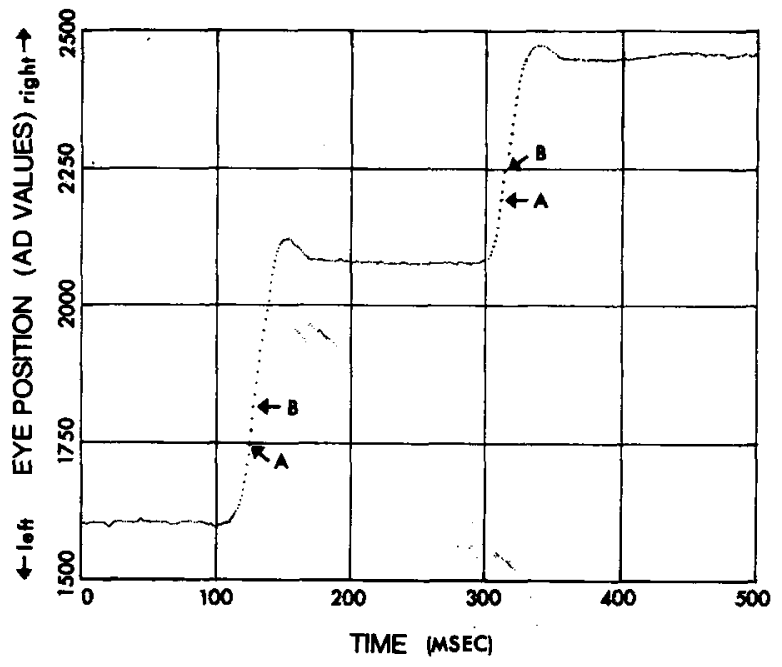

Figure 2. Time required for a display change to be made. The point marked $A$ is the point at which the computer initiated a display change; at the point marked $B, 3$ msec later, the change was realized on the CRT. The vertical axis represents eye position on a scale in which an eye movement of one letter position $\left(1 / 3^{\circ}\right)$ corresponds to a change of about 50 values.

The eight subjects provided about 250 instances in which a display change occurred. This was somewhat smaller than the 280 possible (and was variable among conditions) because subjects sometimes made fewer than six or seven forward saccades of sufficient magnitude in reading a line, and thus missed one of the planned display changes.

To see the effects of display changes on fixations and saccades immediately following the change, Conditions $A$ and $B$ in Table 1 can be compared. Condition A included these data from all lines on which the display changes occurred, and Condition B included corresponding data points for those lines in which no change occurred. It can be seen that the display change produced no effect on the duration of fixations, average length of saccades $[\mathrm{t}(361)=1.2$, p > .10], or number of regressions occurring.

To see the effects of display changes on other fixations and saccades on the line, Conditions $C$ and $\mathrm{D}$ in Table 1 can be compared. Condition $\mathrm{C}$ includes the data for the second, fourth, and sixth fixations and saccades when display changes occurred on the third, fifth, and seventh saccades, and vice versa. Condition $D$ includes data points identified in the same manner, but from lines in which no display change occurred. Again, there are no differences in fixation durations, saccade lengths, or number of regressions between lines in which display changes occurred and those in which they did not.

There is a difference between the values in Conditions $A$ and $B$ vs. those in Conditions $C$ and $D$. This apparently relates to the way the data were selected for these different conditions. In Conditions $A$ and $B$, the data came from those fixations and saccades immediately following a saccade identified as a candidate for a display change. Thus, they followed forward saccades of at least 3.5 letter positions in length. The data in Conditions $C$ and $D$ were selected in a slightly different manner. On lines where the second, fourth, and sixth saccades were identified for potential display changes, data for Conditions $\mathrm{C}$ and $\mathrm{D}$ were taken from the fixations and saccades immediately following those used for data in Conditions A and B. When the third, fifth, and seventh saccades were identified for potential display changes, data for Conditions $\mathrm{C}$ and $\mathrm{D}$ were taken from the fixations and saccades immediately preceding those used for data in Conditions A and B. Thus, those data could be taken from fixations and saccades which followed short saccades and regressive movements. Since our equipment is very sensitive in detecting short saccades of considerably less than one letter position in length $\left(1 / 3^{\circ}\right)$, some of the data in Conditions $\mathrm{C}$ and $\mathrm{D}$ were from fixations and saccades immediately following very small saccades. It appears that fixations following short saccades and regressions may have shorter durations, and are less likely to be followed by regressive movements. Finally, this data selection procedure led to many of the saccades contributing data to Condition $\mathrm{C}$ being the very saccades on which display changes occurred. This apparently had no effect, since the data did not differ between Condition $\mathrm{C}$, in which display changes did occur, and Condition $\mathrm{D}$, in which they did not.

In summary, there is no evidence that display changes of the type occurring in this study produced

Table 1

Eye Movement Data Following Selected Saccades

\begin{tabular}{|c|c|c|c|c|c|c|c|}
\hline \multirow[b]{2}{*}{ Condition } & \multicolumn{3}{|c|}{ Fixation Duration } & \multicolumn{3}{|c|}{ Forward Saccade Length } & \multirow{2}{*}{$\begin{array}{c}\text { Regressions } \\
\text { Total } \\
\text { Number }\end{array}$} \\
\hline & $\mathbf{N}$ & $\begin{array}{c}\text { Average } \\
\text { Duration }\end{array}$ & $\underline{\text { SD }}$ & $\mathbf{N}$ & $\begin{array}{c}\text { Average } \\
\text { Length }\end{array}$ & SD & \\
\hline \multicolumn{8}{|c|}{ Data From Fixation and Saccade Immediately Following a Saccade Identified for Potential Case Change } \\
\hline $\begin{array}{l}\text { (A) Case Change Occurred } \\
\text { (B) No Case Change Occurred }\end{array}$ & $\begin{array}{l}232 \\
247\end{array}$ & $\begin{array}{l}261.3 \\
262.8\end{array}$ & $\begin{array}{l}96 \\
85\end{array}$ & $\begin{array}{l}180 \\
183\end{array}$ & $\begin{array}{l}7.9 \\
7.5\end{array}$ & $\begin{array}{l}3.0 \\
2.9\end{array}$ & $\begin{array}{l}52 \\
64\end{array}$ \\
\hline \multicolumn{8}{|c|}{ Data For Fixations and Saccades Not Immediately Following a Saccade Identified for Potential Case Change } \\
\hline $\begin{array}{l}\text { (C) Line with Case Change } \\
\text { (D) Line With No Case Change }\end{array}$ & $\begin{array}{l}234 \\
250\end{array}$ & $\begin{array}{l}247.9 \\
248.7\end{array}$ & $\begin{array}{l}90 \\
83\end{array}$ & $\begin{array}{l}205 \\
216\end{array}$ & $\begin{array}{l}8.6 \\
8.5\end{array}$ & $\begin{array}{l}4.5 \\
3.7\end{array}$ & $\begin{array}{l}29 \\
34\end{array}$ \\
\hline
\end{tabular}

Note-Fixation durations are in milliseconds; saccade lengths are in letter positions. 
any changes in the eye-movement patterns of the readers. In fact, in most instances the data patterns for lines with and without display changes were very similar indeed. Thus, there is no evidence that changing the visual shape of the letters from one fixation to the next had any disruptive effect on reading. There is some evidence that fixations and saccades following forward saccades of at least 3.5 letter positions in length show different patterns than those following shorter saccades and/or regressive movements, but the nature of this difference was not systematically explored.

\section{DISCUSSION}

In this study, two aspects of the text were changed from one fixation to the next: the visual features of the individual letters and the overall shape, or "envelope," of the words. This was done without changing the semantics, syntax, or orthography of the text. If, in order to allow continuous, smooth reading, it were necessary to integrate such purely visual information as visual features of individual letters and of word shapes across fixations, as a visual buffer model would suggest, then the types of display changes produced in this experiment should have been quite disruptive to the reading process. However, the eye-movement data provided no evidence for any sort of disruption. In addition, the subjects were quizzed following their reading to see if they noticed any irregularities as they read. None reported having noticed anything irregular. The authors themselves also examined the passages under conditions in which the letters were changing case on every forward saccade, and were totally unable to detect that any stimulus change was occurring. The only way we could tell that changes were taking place was to specifically remember the case of a particular letter (the " $T$ " in "ThE" was capitalized), and then to return later and see that it was now lowercase. The display changes were very obvious to onlookers, of course, for whom the changes were usually occurring during fixations rather than during eye movements.

The fact that changing visual shapes of letters and words from one fixation to the next has no apparent effect on reading stands as evidence that such visual data are not being integrated across fixations. This, of course, does not indicate that they are not being used in reading. It simply shows that the visual information used in the reading process is that which is available from the retina during a fixation. Thus, the visual information that permits us to distinguish between a and $A, g$ and $G$, or between LeTtEr and IEtTeR is available only from present retinal stimulation; it is not carried across from fixation to fixation as a means of maintaining visual continuity.

At the same time, it is clear that information at some level of abstraction is being carried from one fixation to the next. As mentioned earlier, Rayner
(1975) reported that changing the contents of a word location in a passage from one fixation to the next did have an effect on the readers, as seen in their eye-movement patterns. The change produced a disrupting effect on reading. Rayner (1978) and Rayner, McConkie, and Ehrlich (in press) also report that the presentation of a word in parafoveal vision reduces the naming time of that word when the person then brings it into foveal vision on the next fixation. The results of the present study suggest that these interference and facilitation effects must be due, not to the carry-over of purely visual information from one fixation to the next, but rather to the carry-over of information resulting from some deeper encoding of the stimulus. Whether this should be thought of in terms of letter identification, identification of orthographic patterns, semantic encoding, phonemic or articulatory coding, or some combination of these, is a topic for further research.

In fact, we suspect that the concept of "visual integration" is the wrong way to think about what is occurring across fixations in reading. Perhaps the reader is simply accessing the visual detail available at the time it is needed to carry out the language processing involved in reading. When a stimulus change occurs during a saccadic eye movement, the change will be disruptive if the new information which the reader seeks is not in harmony with the processing which has taken place previously. If the changed information is not accessed, or if the nature of the change is such that no disharmony results (i.e., if the information obtained from the prior and new stimuli fit together to produce a reasonable and grammatical reading of the text), then the change will not disrupt the reading and will probably go undetected.

\section{REFERENCES}

Cumming, G. D. Eye movements and visual perception. In E. C. Carterette \& M. Friedman (Eds.), Handbook of perception (Vol. VIII). New York: Academic Press, in press.

McConkie, G. W., \& RAYNer, K. Identifying the span of the effective stimulus in reading: Literature review and theories of reading. In H. Singer \& R. B. Ruddell (Eds.), Theoretical models and processes of reading (2nd ed.) Newark, Del: International Reading Association, 1976.

McConkie, G. W., Zola, D., Wolverton, G. S., \& Burns, D. D. Eye movement contingent display control in studying reading. Behavior Research Methods \& Instrumentation, $1978,10,154-166$.

RAYNER, K. The perceptual span and peripheral cues in reading. Cognitive Psychology, 1975, 7, 65-81.

RAYNer, $K$. Foveal and parafoveal cues in reading. In J. Requin (Ed.), Attention and performance VII. New York: Academic Press, 1978.

Rayner, K., McConkie, G. W., \& Ehruich, S. Eye movements and integrating information over fixations. Journal of Experimental Psychology: Human Perception and Performance, in press.

WooDworTr, R. S. Experimental psychology. New York: Holt, 1938.

(Received for publication August 31, 1978; revision accepted December 1, 1978.) 